Supporting Information

\title{
Structure and Photophysical Properties of Porphyrin-Modified Metal Nanoclusters with Different Chain Lengths
}

\author{
Hiroshi Imahori, ${ }^{*}$ Yukiyasu Kashiwagi, ${ }^{\ddagger}$ Yoshiyuki Endo, ${ }^{\ddagger}$ Takeshi Hanada, ${ }^{\#}$ Yoshinobu \\ Nishimura, ${ }^{\dagger}$ Iwao Yamazaki, ${ }^{* \dagger}$ Yasuyuki Araki, ${ }^{\$}$ Osamu Ito, ${ }^{* \$}$ and Shunichi Fukuzumi*,"
}

Department of Molecular Engineering, Graduate School of Engineering, Kyoto University, PRESTO, JAPAN Science and Technology Corporation (JST), Katsura, Nishikyo-ku, Kyoto 615-8510, Japan and Fukui Institute for Fundamental Chemistry, Kyoto University, 34-4, Takano-Nishihiraki-cho, Sakyo-ku, Kyoto 606-8103, Japan

${ }^{*}$ Department of Material and Life Science, Graduate School of Engineering, Osaka University, CREST, JAPAN Science and Technology Corporation (JST), Suita, Osaka 565-0871, Japan \#The Institute of Scientific and Industrial Research, Osaka University, 8-1 Mihoga-oka, Ibaraki, Osaka 567-0047, Japan

'Department of Molecular Chemistry, Graduate School of Engineering, Hokkaido University, Sapporo 060-8628, Japan

${ }^{\$}$ Institute for Multidisciplinary Research for Advanced Materials, Tohoku University, CREST, JST, Katahira, Aoba-ku, Sendai 980-8577, Japan

E-mail: imahori@scl.kyoto-u.ac.jp; yamiw@eng.hokudai.ac.jp; ito@tagen.tohoku.ac.jp; fukuzumi@ap.chem.eng.osaka-u.ac.jp 


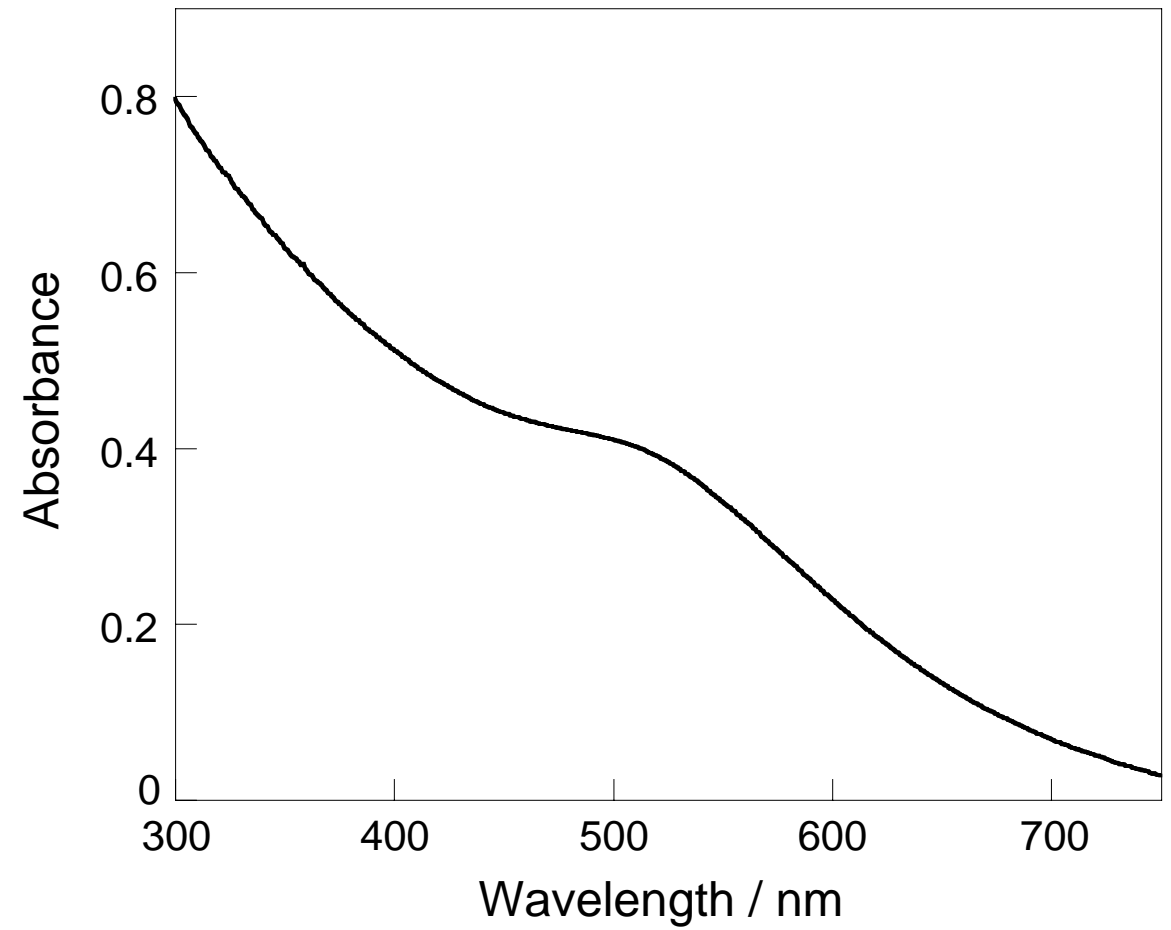

SI 1. UV-vis absorption spectrum of C8AuC $\left(1.0 \times 10^{-5} \mathrm{M}\right)$ in benzene. 


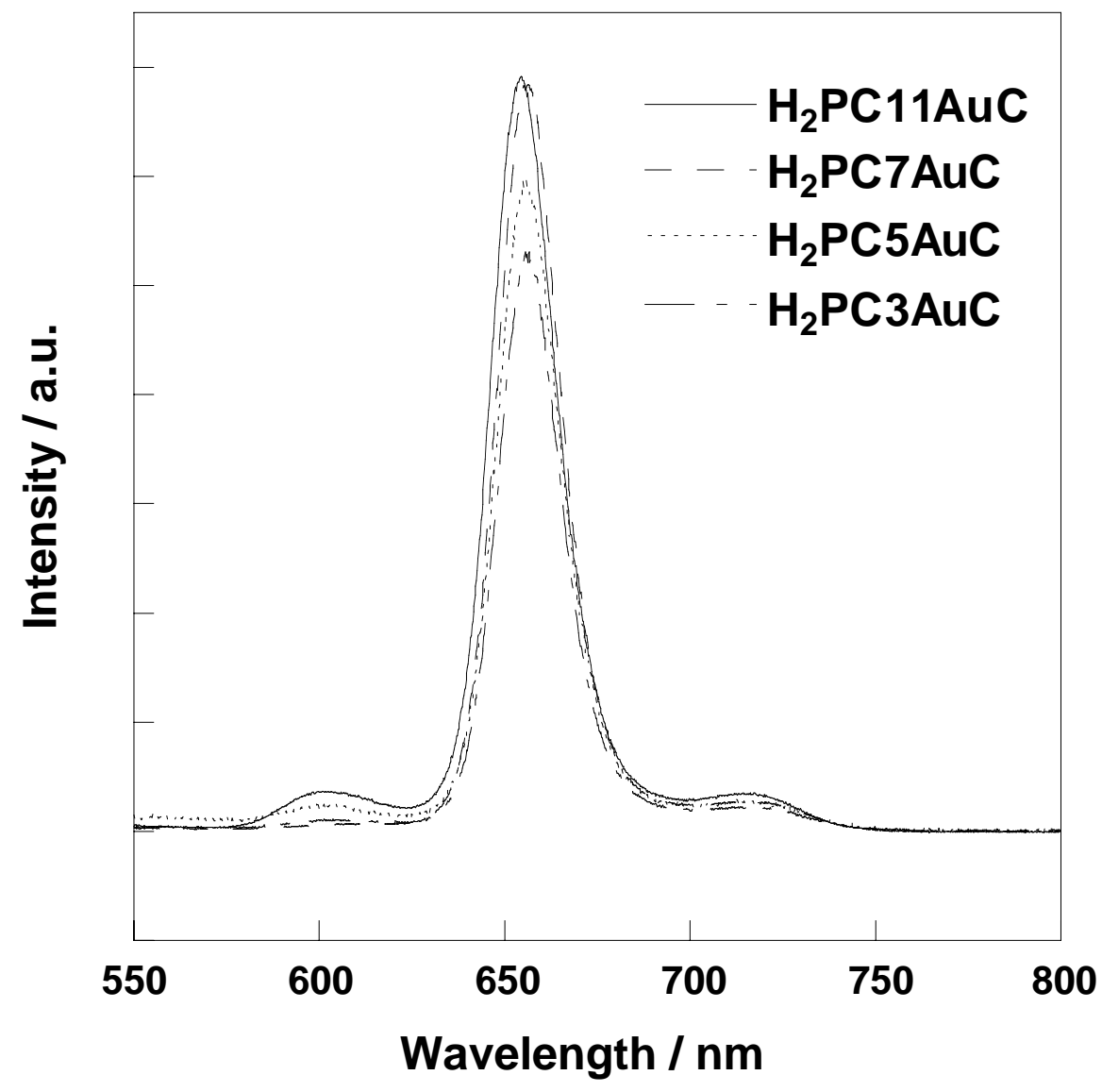

SI 2. Steady-state fluorescence spectra of $\mathbf{H}_{2} \mathbf{P C 1 1 A u C}$ (solid line), $\mathbf{H}_{2} \mathbf{P C 7 A u C}$ (dashed line), $\mathbf{H}_{2} \mathbf{P C 5 A u C}$ (dotted line), and $\mathbf{H}_{2} \mathbf{P C 3 A u C}$ (dashed and dotted line) in benzene. The spectra were measured with the excitation of the Soret band where the absorbance at the Soret peak was adjusted to be identical (1.0). 


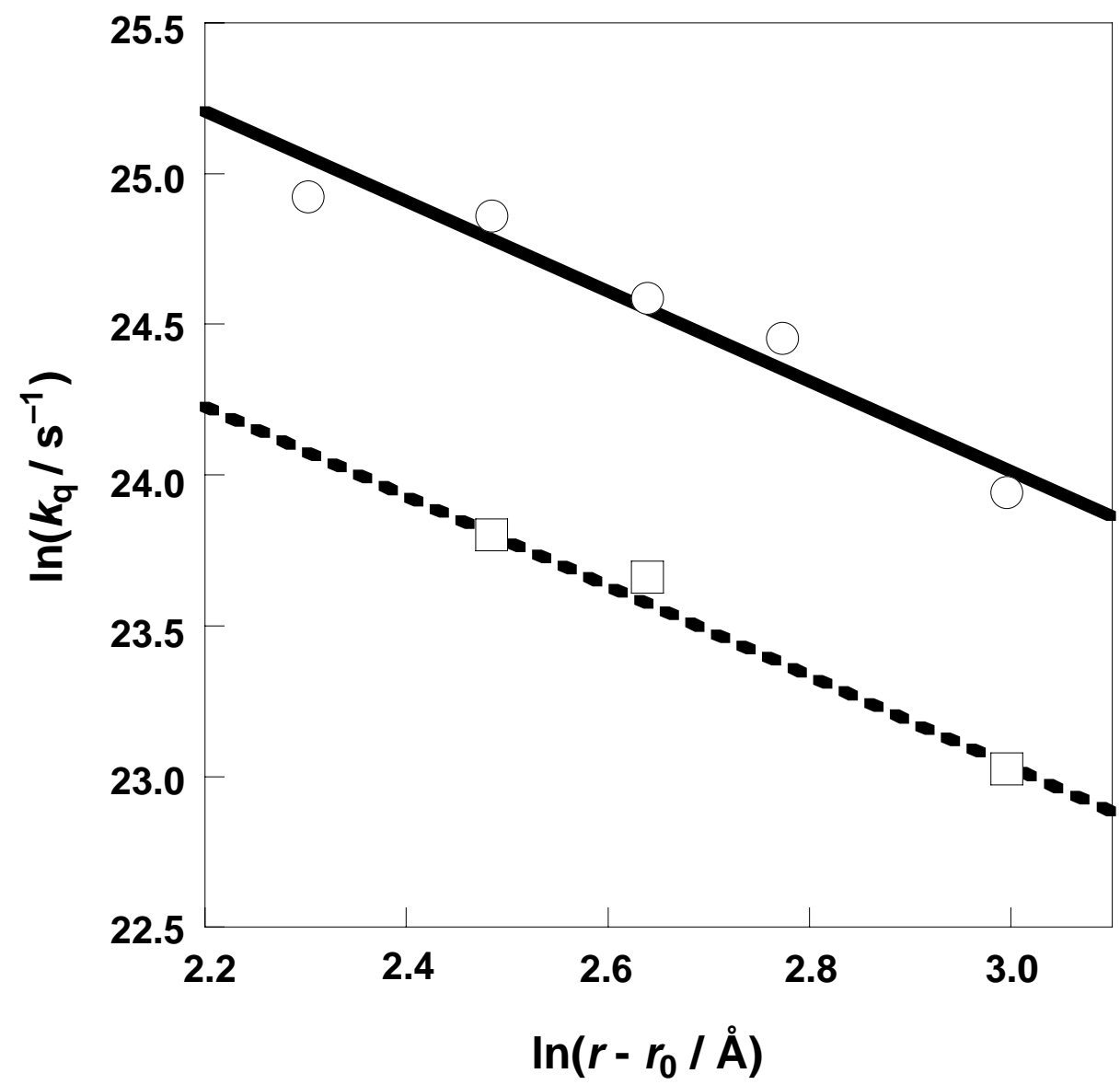

SI 3. Distance dependence of quenching rate constant for $3 \mathrm{D}$ porphyrin MPCs (dashed line with squares) and 2D porphyrin SAMs (solid line with circles). Plots of $\ln \left(k_{\mathrm{q}}\right)$ vs $\ln \left(r-r_{0}\right)$ gave straight lines with the slope for 3D system $(-1.5 \pm 0.1)$ and $2 \mathrm{D}$ system $(1.5 \pm 0.1)$ according to eq 1 . 